\title{
Wave transport in two-dimensional random media: The ballistic to diffusive transition and the extrapolation length
}

\author{
Yun Lai, Sai-Kit Cheung, and Zhao-Qing Zhang* \\ Department of Physics, Hong Kong University of Science and Technology, Clear Water Bay, Kowloon, Hong Kong, China
}

(Received 19 April 2005; published 13 September 2005)

\begin{abstract}
By using a first-principles approach based on the Bethe-Salpeter equation, we study the behavior of wave propagation through a two-dimensional random slab as a function of thickness, $L$, in the region where $L$ is much smaller than the localization length. A general two-dimensional vertex function for the ladder diagrams is derived from the Ward identity. We calculate both the static and the time-resolved transmitted intensities as functions of $L / l$, where $l$ is the mean free path. When $L$ is comparable to $l$, we study the ballistic to diffusive transition. A sharp crossover is observed when $L_{c} \cong 6 l$, which is significantly larger than the crossover thickness of $L_{c} \cong 3 l$ found in three dimensions. When $L \gg l$, we obtain the extrapolation length in two dimensions, i.e., $z_{e}^{2 D} \cong 0.82 l$, which is noticeably larger than the previously used value of $z_{e}^{2 D}=\pi l / 4$ obtained by an analytical approach.
\end{abstract}

DOI: $10.1103 /$ PhysRevE.72.036606

PACS number(s): 42.25.Bs, 46.65.+g, 43.20.+g

\section{INTRODUCTION}

The wave transport in random media is known to acquire diffusive behavior due to multiple scattering [1]. Many wave phenomena in strongly scattering materials, including coherent backscattering, continuous-wave transmission, pulse propagation, and speckle correlations, are well described by the diffusion approximation [2-5]. Despite the success and widespread use of this simple approximation, limitations exist. One such limitation occurs in thin samples, where the number of scatterings is insufficient to randomize the phases and directions of the emerging waves and a crossover to ballistic transport will occur. In the past, many works have been devoted to this crossover behavior both experimentally and theoretically and the particular interest was on the crossover thickness below which the diffusion approximation becomes invalid [6-17]. Most of these works were done for three-dimensional (3D) systems and different answers were found depending on the physical quantities measured. For examples, the steady state photon transmission measurements [3] and diffusing wave spectroscopy (DWS) experiment [6] have indicated that the transport of photons is diffusive for sample thickness, $L$, as low as 3-5 mean free paths, $l$. In contrast, pulsed optical transmission measurements [7-9] have reported systematic deviations from diffusion theory at much larger values of $L / l \leqslant 8-10$. Recently, ultrasonic pulse transmission experiments in strongly scattering media consisting of glass beads immersed in water have been carried out and, at the same time, first-principles calculations of both the frequency correlation function and the time-domain profile of the transmitted intensity have been performed by solving the ladder approximation of the BetheSalpeter equation [14]. Both theory and experiments have observed an abrupt crossover between ballistic and diffusive behavior in the peak arrival time after a pulsed excitation

\footnotetext{
*Corresponding author. Fax: 852-2358-1652; Email address: phzzhang@ust.hk
}

when $L_{c} / l \cong 3$. Waves transport through thin slabs of random media with internal reflection has also been discussed [15]. The crossover from ballistic to diffusive behavior was found to occur at $L_{c} / l \cong 3$ independent of the presence of internal reflection. However, when $L / l>3$, an anomalous diffusion region has been found, in which the diffusion constant increases with decreasing $L$. For two dimensions, Tourin et al. $[16,17]$ have carried out ultrasonic experiments to investigate the ballistic and diffusive waves in a multiple scattering medium consisting of randomly placed steel rods in water background. They concluded that the ballistic and diffusive parts can coexist in a large range of $L / l$, indicating a large value of $L_{c} / l$. However, the precise value of $L_{c} / l$ was not reported. This may be due to the presence of resonance in the pulse spectrum. In this case, the mean free path becomes frequency dependent and is peaked at the resonant frequency. An averaging process weighted by the spectrum content is required to obtain an averaged mean free path. However, the frequency content of the transmitted wave becomes time dependent. This makes the definition of an averaged mean free path ambiguous.

Besides the ballistic to diffusive transition, the extrapolation length, $z_{e}$, is an interesting and useful quantity which is also not well-known in two dimensions. In the diffusive regime, the diffusion approximation works well. However, when the ratio of $L / l$ is not very large, wave transport can be sensitive to the value of $z_{e}$ used in the boundary condition of the diffusion equation and, therefore, an accurate value of $z_{e}$ is necessary. In $3 \mathrm{D}$, the value of $z_{e}^{3 D}$ has been accurately determined from the Milne solution as $z_{e}^{3 D} / l \cong 0.7104$ [18]. This ratio is greater than the value of $2 / 3$ obtained by using an analytical approach based on the flux consideration at the sample boundary [19]. By using the same approach in 2D, it can be shown that $z_{e}^{2 D} / l=\pi / 4$. This number has been used in the literature [20]. However, the correct value of $z_{e}^{2 D}$ in $2 \mathrm{D}$ should also be obtained from the solution of the energy transport equation in $2 \mathrm{D}$, which may also yield a value greater than $\pi / 4$.

In this work, we study both the ballistic to diffusive transition and the extrapolation length in 2D. For these purposes, 
we calculate both the static and the time-resolved transmitted intensities of waves passing through a two-dimensional random slab as a function of slab thickness, $L$, by using a firstprinciples approach based on the Bethe-Salpeter equation. To ensure flux conservation, we derive a dynamic $2 \mathrm{D}$ vertex function for the ladder diagrams based on the Ward identity. In the static limit and the limit of large $k l$, where $k$ is the wave vector, our vertex function recovers the previously known result. When $L$ is comparable to $l$, we find a sharp crossover from ballistic to diffusive behavior when $L_{c} \cong 6 l$, which is significantly larger than the crossover thickness of $L_{c} \cong 3 l$ found in $3 \mathrm{D}$. In the case of large $L / l$, we also investigate the extrapolation length. We find $z_{e}^{2 D} \cong 0.82 l$, which is noticeably larger than $\pi l / 4$ obtained analytically. It should be pointed out that since all waves in 2D random media are believed to be localized [21] and the localization length is predicted as $\xi \simeq l \exp (\pi k l / 2)$ [1,22], in our calculations, we limit ourselves to the region of $k l \geqslant 2 \pi$ and $L / l \leqslant 40$ so that $L / \xi<0.002$. In this case the wave interference effects are believed to be negligible. The diffusion behavior in 2D $[16,17,23]$ and 1D [24] has also been studied both experimentally and numerically in the past.

\section{THEORY}

Consider a plane-wave pulse normally incident on the front surface $(z=0)$ of a slab of thickness $L(0<z<L)$ containing random scatterers. At time $t$ and position $\vec{r}$, the field is specified by the time-dependent wave function $\psi(t, \vec{r})$, which can be written as

$$
\psi(t, \vec{r})=(2 \pi)^{-1} \int d \Omega \exp (-i \Omega t) f(\Omega) \phi_{\Omega}(\vec{r}),
$$

where $f(\Omega)$ describes the spectral content of the pulse, and $\phi_{\Omega}(\vec{r})$ is the spatial part of the wave function. In order to obtain useful physical information, we have to consider the ensemble-averaged quantities. The ensemble-averaged intensity corresponding to Eq. (1) can be expressed as

$$
\begin{aligned}
\left\langle|\psi(t, \vec{r})|^{2}\right\rangle= & (2 \pi)^{-2} \int d \Omega\left[\int d \omega f\left(\Omega^{+}\right) f^{*}\left(\Omega^{-}\right)\right. \\
& \left.\times\left\langle\phi_{\Omega^{+}}(\vec{r}) \phi_{\Omega^{-}}^{*}(\vec{r})\right\rangle \exp (-i \omega t)\right],
\end{aligned}
$$

where \langle\rangle denotes configurational averaging, and the frequencies $\Omega^{ \pm}=\Omega \pm(\omega / 2)$ can be written in terms of the center frequency $\Omega$ and the modulation frequency $\omega$. Here we have followed the notations used in Ref. [14] in the study of the ballistic to diffusive transition in 3D. In the literature, $\Omega$ has been often used to denote the modulation frequency and $\omega$ for the center frequency. From Eq. (2), it is clear that the ensemble-averaged intensity $\left\langle|\psi(t, \vec{r})|^{2}\right\rangle$ is given by the Fourier transform of the frequency correlation function $C_{\Omega}(\omega, \vec{r})=\left\langle\phi_{\Omega^{+}}(\vec{r}) \phi_{\Omega^{-}}^{*}(\vec{r})\right\rangle$, which is the fundamental quantity to be determined. In fact, the frequency correlation function $C_{\Omega}(\omega, \vec{r})$ is a special case for the space-frequency correlation function $\widetilde{C}_{\Omega}\left(\omega ; \vec{r}, \vec{r}^{\prime}\right)=\left\langle\phi_{\Omega^{+}}(\vec{r}) \phi_{\Omega^{-}}^{*}\left(\vec{r}^{\prime}\right)\right\rangle$, which can be ob- tained from the Bethe-Salpeter equation $[1,14]$

$$
\begin{aligned}
\widetilde{C}_{\Omega^{\prime}}\left(\omega ; \vec{r}, \vec{r}^{\prime}\right)= & \left\langle\phi_{\Omega^{+}}(\vec{r})\right\rangle\left\langle\phi_{\Omega^{-}}^{*}\left(\vec{r}^{\prime}\right)\right\rangle \\
& +\int d \vec{r}_{1} d \vec{r}_{2} d \vec{r}_{3} d \vec{r}_{4}\left\langle G_{\Omega^{+}}\left(\vec{r}, \vec{r}_{1}\right)\right\rangle \\
& \times\left\langle G_{\Omega^{-}}^{*}\left(\vec{r}^{\prime}, \vec{r}_{2}\right)\right\rangle U\left(\vec{r}_{1}, \vec{r}_{2}, \vec{r}_{3}, \vec{r}_{4}\right) \widetilde{C}_{\Omega}\left(\omega ; \vec{r}_{3}, \vec{r}_{4}\right) .
\end{aligned}
$$

Here $U\left(\vec{r}_{1}, \vec{r}_{2} ; \vec{r}_{3}, \vec{r}_{4}\right)$ is the irreducible vertex function. $\left\langle G_{\Omega^{+}}\left(\vec{r}, \vec{r}_{1}\right)\right\rangle$ and $\left\langle G_{\Omega^{-}}\left(\vec{r}^{\prime}, \vec{r}_{2}\right)\right\rangle$ are the ensemble-averaged Green's functions, which describe, respectively, the coherent part of wave propagation from $\vec{r}_{1}$ to $\vec{r}$, and from $\vec{r}_{2}$ to $\vec{r}^{\prime}$. Under a plane wave incidence, the ensemble-averaged field $\left\langle\phi_{\Omega^{ \pm}}(\vec{r})\right\rangle$ takes the form of $\exp \left(i \Omega^{ \pm} z / v-z / 2 l\right)$, where $l$ is the scattering mean free path and $v$ is the phase velocity in the slab. Since $\left\langle\phi_{\Omega^{ \pm}}\right\rangle$represents the coherent part of the waves inside the random slab, $v$ should be considered as the effective medium phase velocity of the random slab [14]. Since for our purpose only frequency correlation function $C_{\Omega}(\omega, \vec{r})$ is needed, we let $\vec{r}^{\prime}=\vec{r}$ in Eq. (3). The ensemble-averaged Green's function in Eq. (3) has the form of Hankle function in $2 \mathrm{D}$, i.e.,

$$
\left\langle G_{\Omega^{ \pm}}\left(\vec{r}, \vec{r}_{1,2}\right)\right\rangle=-\frac{i}{4} H_{0}^{(1)}\left(K^{ \pm}\left|\vec{r}-\vec{r}_{1,2}\right|\right),
$$

where $K^{ \pm}=\Omega^{ \pm} / v+i / 2 l$ are complex wave vectors. In this work, for simplicity, we assume the scatterings are pointlike and isotropic so that $l$ is also the transport mean free path. In the lowest order approximation, we take $l=1 / n \sigma$ as the bare mean free path, where $n$ is the number density and $\sigma$ is the total scattering cross section. This approximation corresponds to the ladder approximation in the vertex function $U$ as required by Ward identity, which takes the following form [1]:

$$
\Sigma^{+}\left(\Omega^{+}\right)-\Sigma^{-}\left(\Omega^{-}\right)=\left[\left\langle G^{+}\left(\Omega^{+}, \vec{r}=\vec{r}^{\prime}\right)\right\rangle-\left\langle G^{-}\left(\Omega^{-}, \vec{r}=\vec{r}^{\prime}\right)\right\rangle\right] U,
$$

where $\Sigma^{+}$and $\Sigma^{-}$are the self-energies of $\left\langle G^{+}\right\rangle$and $\left\langle G^{-}\right\rangle$. The left-hand side of Eq. (5) can be expressed as

$$
\Sigma^{+}\left(\Omega^{+}\right)-\Sigma^{-}\left(\Omega^{-}\right)=-\frac{2 \Omega}{l v} i .
$$

The vertex function $U$ is thus obtained as

$$
U(\Omega, \omega)=\frac{-\frac{2 \Omega}{l v} i}{\left\langle G^{+}\left(\Omega^{+}, \vec{r}=\vec{r}^{\prime}\right)\right\rangle-\left\langle G^{-}\left(\Omega^{-}, \vec{r}=\vec{r}^{\prime}\right)\right\rangle} .
$$

Equation (7) is valid in any dimension. For the 3D case, $\left\langle G^{+}\left(\Omega^{+}, \vec{r}=\vec{r}^{\prime}\right)\right\rangle-\left\langle G^{-}\left(\Omega^{-}, \vec{r}=\vec{r}^{\prime}\right)\right\rangle=-2 \Omega / 4 \pi v i$, which leads to the commonly used vertex function in $3 \mathrm{D}$, i.e., $U_{3 D}=4 \pi / l$ $[1,14,25]$. We note that $U_{3 D}$ is independent of $\Omega$ and $\omega$. However, in 2D we have 


$$
\begin{aligned}
\left\langle G^{+}\left(\Omega^{+}, \vec{r}=\vec{r}^{\prime}\right)\right\rangle-\left\langle G^{-}\left(\Omega^{-}, \vec{r}=\vec{r}^{\prime}\right)\right\rangle & \\
= & -\left.\frac{i}{4} H_{0}^{(1)}\left(K^{+} r\right)\right|_{r \rightarrow 0}-\left.\frac{i}{4} H_{0}^{(1) *}\left(K^{-} r\right)\right|_{r \rightarrow 0} \\
& =\frac{\ln \left(\frac{\left|K^{+}\right|}{\left|K^{-}\right|}\right)}{2 \pi}+\frac{i\left(\theta^{+}+\theta^{-}-\pi\right)}{2 \pi},
\end{aligned}
$$

where $\theta^{+}=\ln \left(K^{+} /\left|K^{+}\right|\right) / i, \quad \theta^{-}=\ln \left(K^{-} /\left|K^{-}\right|\right) / i$. Substituting back in Eq. (7), we obtain

$$
U_{2 D}=\frac{\frac{-4 \pi \Omega i}{l v}}{\ln \left(\frac{\left|K^{+}\right|}{\left|K^{-}\right|}\right)+i\left(\theta^{+}+\theta^{-}-\pi\right)} .
$$

Different from the $3 \mathrm{D}$ case, the vertex function in $2 \mathrm{D}$ depends on both $\Omega$ and $\omega$. It possesses the following symmetry properties: $\quad U_{2 D}(-\Omega, \omega)=U_{2 D}(\Omega, \omega) \quad$ and $\quad U_{2 D}(\Omega,-\omega)$ $=U_{2 D}^{*}(\Omega, \omega)$. At $\omega=0$ and $\Omega>0$, Eq. (9) reduces to

$$
U_{2 D}(\omega=0)=\frac{\frac{\Omega}{l v}}{\frac{1}{4}-\frac{\theta}{2 \pi}}, \quad \theta=\arctan \left(\frac{v}{2 l \Omega}\right) .
$$

In the limit of $\Omega l / v=k l \gg 1$, Eq. (10) recovers the previously used vertex function $U_{2 D}=4 k / l=4 \Omega / v l$ in $2 \mathrm{D}$ [26]. Thus the vertex function shown in Eq. (9) is more general and should be used in the study of wave dynamics, particularly when $k l$ is not very large. We will show later that even when $\mathrm{kl}$ $\approx 60$, the use of $U_{2 D}=4 \mathrm{k} / \mathrm{l}$ will give erroneous results, due to violation of flux conservation.

The vertex function in real space is thus obtained as $U\left(\vec{r}_{1}, \vec{r}_{3} ; \vec{r}_{2}, \vec{r}_{4}\right)=U_{2 D} \delta\left(\vec{r}_{1}-\vec{r}_{3}\right) \delta\left(\vec{r}_{1}-\vec{r}_{2}\right) \delta\left(\vec{r}_{3}-\vec{r}_{4}\right)$. With this form of $U\left(\vec{r}_{1}, \vec{r}_{3} ; \vec{r}_{2}, \vec{r}_{4}\right)$, Eq. (3) generates a sum of ladder diagrams, which represents the multiple scattering of waves without interferences of different paths, and turns into the well-known radiative transfer equation. Due to the symmetry in our problem, $C_{\Omega}(\omega, \vec{r})$ is only dependent on the $z$ coordinate. Thus we have

$$
C_{\Omega}(\omega, z)=\exp \left(\frac{i \omega z}{v}-\frac{z}{l}\right)+\frac{U_{2 D}}{16} \int d z_{1} C_{\Omega}\left(\omega, z_{1}\right) H\left(z-z_{1}\right)
$$

where

$$
\begin{aligned}
& H\left(z-z_{1}\right) \\
& \quad=\int d y_{1} H_{0}^{(1)}\left(K^{+} \sqrt{y_{1}^{2}+\left(z-z_{1}\right)^{2}}\right) H_{0}^{(1) *}\left(K^{-} \sqrt{y_{1}^{2}+\left(z-z_{1}\right)^{2}}\right) .
\end{aligned}
$$

The first term in Eq. (11) represents the coherent contribution, while the second term describes the scattered wave contribution. Equation (11) takes a similar form of the Milne equation and can be solved numerically to obtain the frequency correlation function $C_{\Omega}(\omega, z)$ as a function of $z, \Omega$,

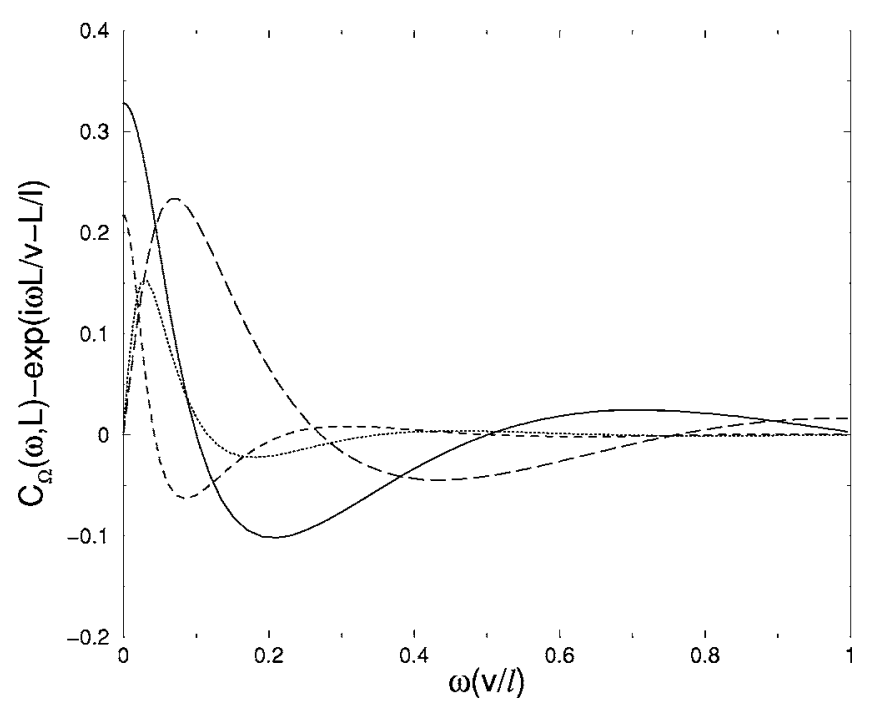

FIG. 1. Correlation function $C_{\Omega}(\omega, L)-\exp (i \omega L / v-L / l)$ as a function of $\omega$ for $L=6 l$ (solid line: real part; long dashed line: imaginary part) and $L=10 l$ (dashed line: real part; dotted line: imaginary part), with $k l=20 \pi$.

and $\omega$, given a set of parameters $v, l$, and $L$. After $C_{\Omega}(\omega, z)$ is solved, we can substitute it back into Eq. (2) to calculate the ensemble-averaged intensity $\left\langle|\psi(t, z)|^{2}\right\rangle$. It should be pointed out that Eqs. (11) and (12) exhibit the $\Omega$ dependence in both the functions $U_{2 D}$ and $H_{0}^{(1)}$. However, the corresponding equations in 3D, i.e., Eqs. (8) and (9) of Ref. [14] do not have $\Omega$ dependence. Thus it is interesting and important to see if our results depend on the choice of $\Omega$. In our calculations below, we choose two different values of $\Omega$, i.e., $k l$ $=2 \pi$ and $k l=20 \pi$. According to $\xi \simeq l \exp (\pi k l / 2)[1,22]$, the corresponding localization lengths are $\xi=1.9 \times 10^{4} l$ and 7.3 $\times 10^{42} l$, which are much larger than the values of $L$ used in our calculations so that the use of ladder diagrams is justified.

\section{NUMERICAL RESULTS AND DISCUSSION}

In order to study the ballistic to diffusive transition, we calculate the time-resolved transmitted intensity $I(L, t)$ at the output surface at different ratios of $L / l$. We first calculate the frequency correlation function $C_{\Omega}(\omega, L)$ by numerically solving Eq. (11). In Fig. 1, we plot the part of the correlation function $\bar{C}_{\Omega}(\omega, L)=C_{\Omega}(\omega, L)-\exp (i \omega L / v-L / l)$ corresponding to the scattered waves, as a function of $\omega$, with center frequency of $k l=20 \pi$ for slabs of thickness $L=6 l$ and $10 l$, respectively. The solid line and the long-dashed line denote the real and imaginary part of $\bar{C}_{\Omega}(\omega, L)$ for $L=6 l$, and the dashed line and dotted line denote the real and imaginary part of $\bar{C}_{\Omega}(\omega, L)$ for $L=10 l$. Similar to the $3 \mathrm{D}$ case, $\bar{C}_{\Omega}(\omega, L)$ is a decreasing and oscillating function of $|\omega|$. When slab thickness $L$ is increased, the envelope of $\bar{C}_{\Omega}(\omega, L)$ becomes narrower. Then, we calculate $I_{\Omega}(L, t)$ $=\int d \omega f\left(\Omega^{+}\right) f^{*}\left(\Omega^{-}\right) \bar{C}_{\Omega}(\omega, L) \exp (-i \omega t)$ by numerical integration, with $f\left(\Omega^{ \pm}\right)=1$ in the envelope range of $\bar{C}_{\Omega}(\omega, L)$. In 


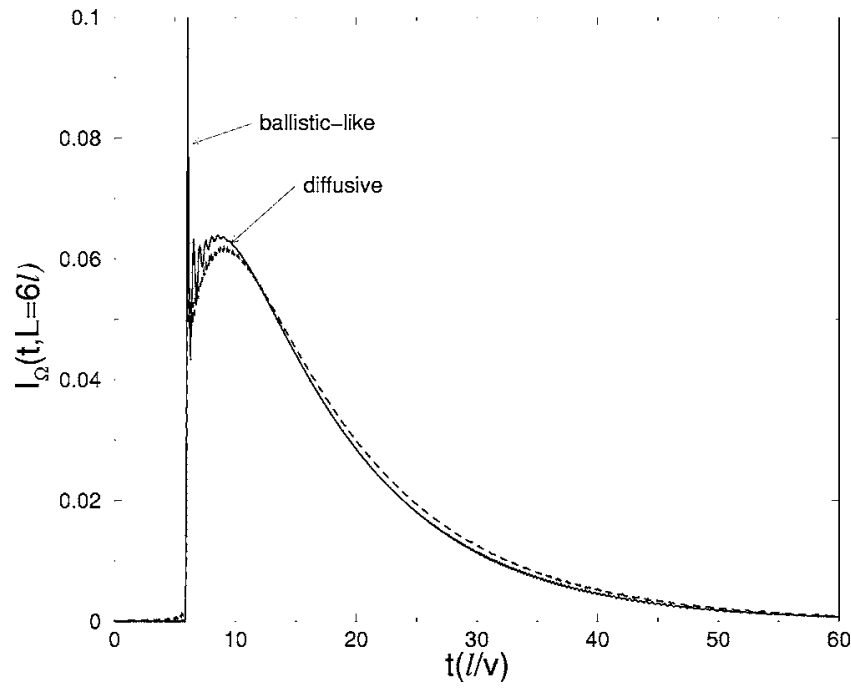

FIG. 2. The solid and dashed lines describe $I_{\Omega}(L=6 l, t)$ for $k l$ $=20 \pi$ and $k l=2 \pi$, respectively.

Fig. 2, the $I_{\Omega}(L, t)$ for a slab of $L=6 l$ with $k l=20 \pi$ and $2 \pi$ are plotted as solid and dashed lines, respectively. We note that the two functions of $I_{\Omega}(L, t)$ are almost identical, although their $\Omega$ 's are ten times different. In fact, this is true for all the quantities we have studied. Thus, in the following, we show only the results of $k l=20 \pi$ and omit the subscript $\Omega$ in $I_{\Omega}(L, t)$. In Fig. 2 we also find that at $L=6 l$, the scattered intensity $I(L, t)$ consists of two peaks in time. One peak is ballistic-like, in the sense that it is narrow and sharp, and arrives at the output surface at the exact time $L / v$. The other peak is due to the diffusive waves, which are scattered in random direction and thus arrive later. The coexistence of ballistic-like peak and the peak of diffusive waves has been found in the time-of-flight experiments [16,17]. Physically, if we consider the multiple scattering process on an effective medium basis, the ballistic-like peak in Fig. 2 corresponds to the part of the scattered waves which are forward scattered along the propagation path in the effective medium of the slab. Since the coherent wave intensity, which is not shown in Fig. 2, decays exponentially as $\exp (-L / l)$, the ballisticlike peak formed by forward scattered waves will overtake the coherent wave intensity and determine the ballisticdiffusive transition.

In Fig. 3, we plot the peak arrival time $t_{p}$ of the scattered intensity as a function of slab thickness $L$ in $\log -\log$ scale. Two linear segments are clearly seen. The one with $L \leqslant 6 l$ has a slope of one, showing the ballistic transport with $t_{p}$ $=L / v$. When $L>6 l$, the other segment shows a slope of two, indicating a diffusive transport with $t_{p} \propto L^{2}$. Thus a sharp crossover is also found in 2D, but with a crossover thickness $L_{c} \cong 6 l$, which is twice that found in $3 \mathrm{D}$. We should mention again that the results of $k l=2 \pi$ are indiscernible from that of $k l=20 \pi$ shown in Fig. 3.

When $L \gg l$, Eq. (11) allows us to study the extrapolation length in 2D. Before presenting our numerical results, let us summarize some known analytical results derived from the diffusion theory. The static diffusion equation takes the form

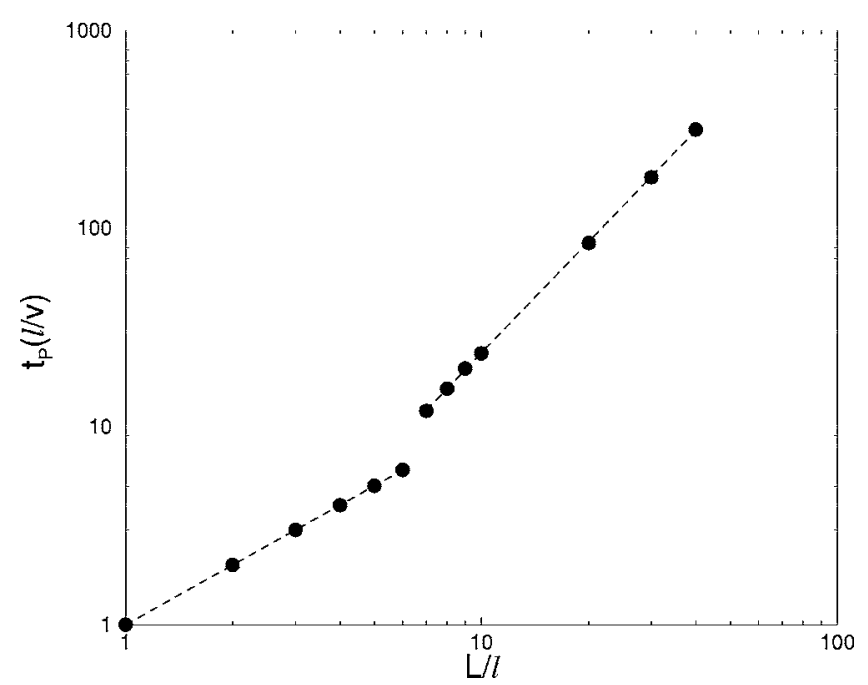

FIG. 3. The peak arrival time $t_{p}$ of scattered intensity $I(L, t)$ as a function of $L$. The data for $L \leqslant 6 l$ and $L>6 l$ are fitted separately as the dashed lines.

$$
-D \nabla^{2} I(z)=\delta\left(z-z_{p}\right),
$$

where $D$ is the diffusion constant, $I(z)$ is the static intensity, and $z_{p}$ represents the penetration length of the incident wave. This equation is solved with the boundary conditions $I\left(-z_{e}\right)$ $=I\left(L+z_{e}\right)=0$, where $z_{e}$ is the extrapolation length to be determined. The solution of Eq. (13) for $z>z_{p}$ is expressed as

$$
I(z)=\frac{\left(z_{p}+z_{e}\right)\left(L+z_{e}-z\right)}{D\left(L+2 z_{e}\right)}, \quad z>z_{p}
$$

Thus $I(z)$ is linearly decaying for $z>z_{p}$ and extrapolating to $z$ axis at $z=L+z_{e}$. At $z=L$, the inverse of the intensity $I(L)$ takes the form

$$
\frac{1}{I(L)}=\frac{D\left(L+2 z_{e}\right)}{\left(z_{p}+z_{e}\right) z_{e}},
$$

which is linearly dependent on $L$ and extrapolating to $L$ axis at $L=-2 z_{e}$. Here, we solve Eq. (11) in the static limit and use the so-obtained $I(z)$ and $I(L)$ in Eqs. (14) and (15), respectively, to determine the extrapolation length $z_{e}^{2 D}$ in $2 \mathrm{D}$.

In the static limit, we use a plane wave incidence containing a single frequency $\Omega$. Thus $\left\langle|\psi(t, z)|^{2}\right\rangle=(2 \pi)^{-2} C_{\Omega}(\omega$ $=0, z)$. In Fig. 4, we plot the scattered wave intensity distributions $I(z)=C_{\Omega}(\omega=0, z)-\exp (-z / l)$ for $L=5 l, 10 l$, and $20 l$, as solid, long-dashed, and dashed lines, respectively, under an incident plane wave with $k l=20 \pi$. When the slab thickness $L$ becomes large enough, an almost linearly decaying region appears inside the slab, indicating domination of diffusive waves in that region. This is seen more clearly in the inset of Fig. 4, which describes the second derivatives of $I(z)$ with respect to $z$. Here we also notice that near the output surface of the slab, the linear decay is lost. So the transmitted intensity $I(L)$ will deviate slightly from the diffusion result. Nevertheless, Eq. (15) provides a convenient way to determine $z_{e}$ [3]. In Fig. 5, we plot the inverse of the intensity at the output surface of the slab, $1 / I(L)$, as a function of slab 


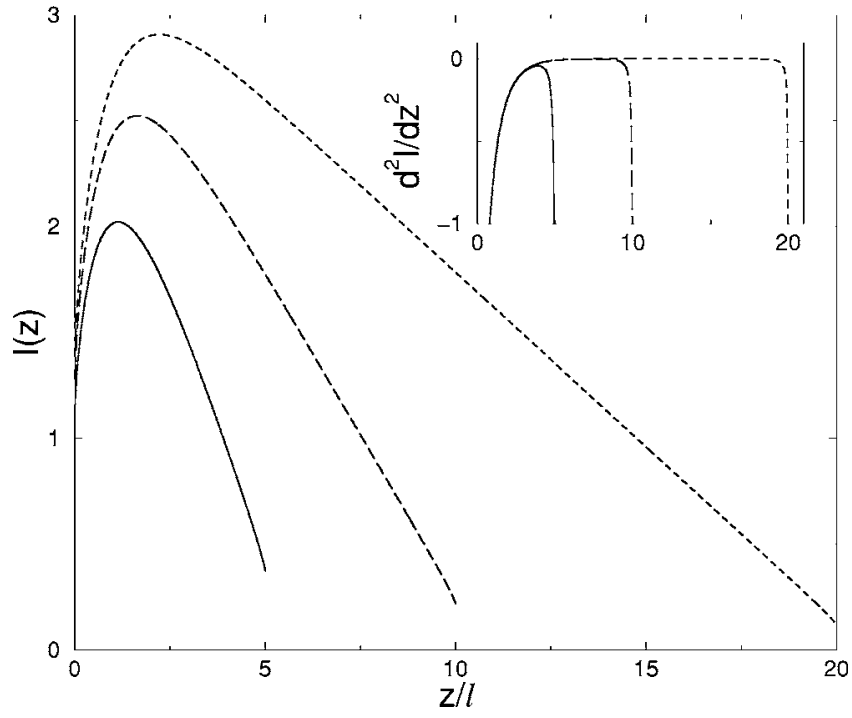

FIG. 4. Scattered wave intensity distribution $I(z)$ under a plane wave incidence of $k l=20 \pi$, for slabs of thickness $L=5 l$ (solid line), $10 l$ (long-dashed line), and $20 l$ (dashed line). The inset graph denotes the corresponding $d^{2} I(z) / d z^{2}$.

thickness $L$ for $k l=20 \pi$. The inset shows the data for $L$ $\leqslant 10 l$. It is seen that $1 / I(L)$ becomes a nearly linear function of $L$ when slab thickness $L>6 l$. By extrapolating the data of $1 / I(L)$ at large $L$ 's to the $L$ axis, we obtain the extrapolation length $z_{e}^{2 D} \cong 0.8184 l$ for $k l=20 \pi$. In Fig. 5, we also plot the results of $1 / I(L)$ calculated by using $U_{2 D}=4 k / l$ by open circles. In this case, $1 / L(l)$ increases exponentially with $L$. Thus it is clear that the use of vertex function $U_{2 D}=4 \mathrm{k} / \mathrm{l}$ violates the flux conservation and gives rise to a behavior of an absorptive medium. For the case of $k l=2 \pi$, we obtain a similar value $z_{e}^{2 D} \cong 0.8206 l$.

As we have mentioned before that $I(z)$ loses its linear decay near the boundary. This might make Eq. (15) invalid.

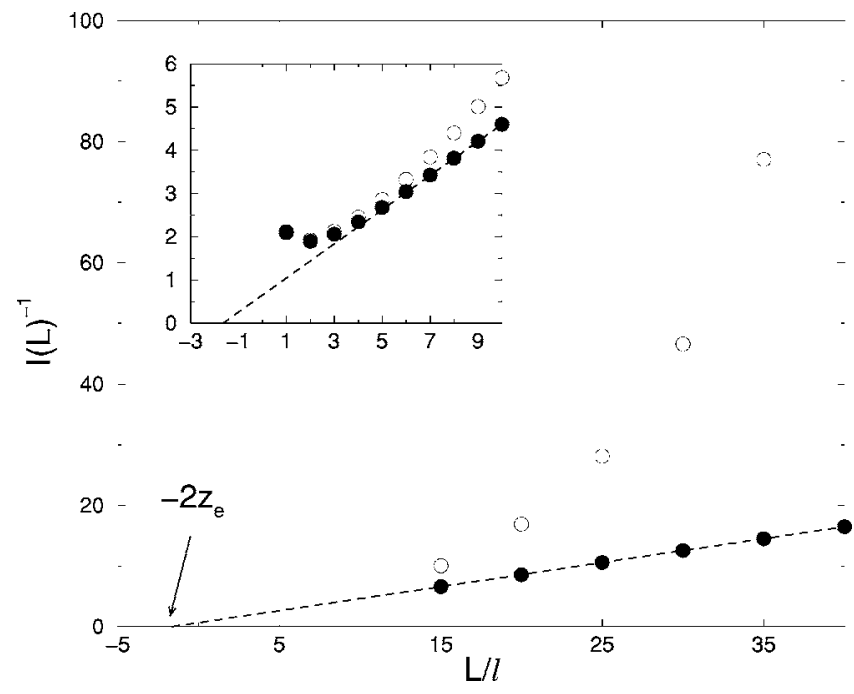

FIG. 5. The inverse of scattered intensity $I(L)^{-1}$ are plotted in closed circles, as a function of the slab thickness $L$, under a plane wave incidence of $k l=20 \pi$. The dashed line is a linear fit for $L$ $\geqslant 15 l$. The open circles denote the result calculated by using the vertex function $U_{2 D}=4 k / l$. The inset graph shows data for $L \leqslant 10 l$.

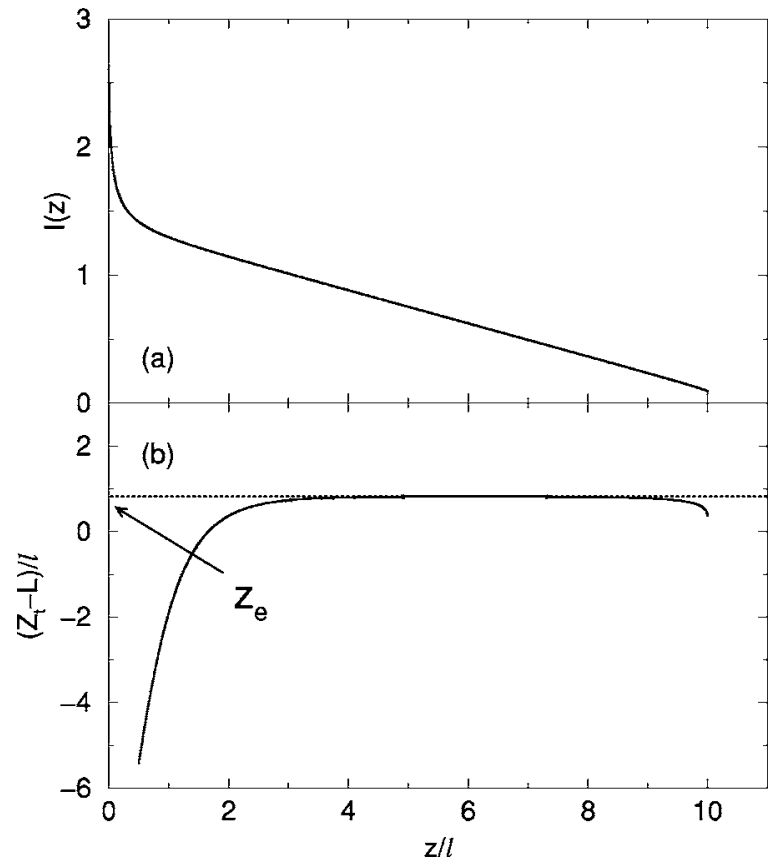

FIG. 6. (a) Intensity distribution $I(z)$ under an approximate point source $\delta(z)$ with $k l=20 \pi$; (b) $z_{t}$ denotes the intersection value of the tangent line of $I(z)$ taken at $z$ and the $z$ axis. $z_{e}=z_{t}-L$ is chosen as the maximum $z_{t}-L$ for $z$ inside the region of diffusive waves.

In order to check this point, we recalculate $z_{e}^{2 D}$ by using Eq. (14). Another disadvantage of the previous calculation is that the coherent source term $\exp (-z / l)$, though decaying exponentially, exists inside the whole slab. In order to minimize the effects of the coherent source, we use a delta functionlike source, i.e.,

$$
\left|\left\langle\psi_{\Omega_{0}}(z)\right\rangle\right|^{2}= \begin{cases}1000 l, & 0<z \leqslant 0.001 l \\ 0, & z>0.001 l\end{cases}
$$

instead of the exponentially decaying source in Eq. (11). Now, in order to obtain the extrapolation length, we take the derivative of $I(z)$ at $z$ as the tangent slope and extrapolate the tangent line to the $z$ axis at $z_{t}$. Then, according to Eq. (14), the extrapolation length can be determined from $z_{e}^{2 D}=z_{t}-L$ if the tangent line is taken at $z$ in the region dominated by diffusive waves, i.e., $I(z)$ is linear around $z$. In Fig. 6(a), we plot the intensity distribution in a slab of $L=10 l$ for a frequency of $k l=20 \pi$. The corresponding $z_{t}-L$ as a function of $z$ is plotted in Fig. 6(b). The flat region indicates the region dominated by diffusive waves. Here we simply choose the maximum $z_{t}-L$ around the center of the flat part as the final extrapolation length. In Fig. 7 we plot the obtained $z_{e}^{2 D}$ at different slab thicknesses for $k l=20 \pi$. It is found that the extrapolation length saturates quickly to $z_{e}^{2 D} \cong 0.8183 l$ as $L$ $>10 l$. For the case of $k l=2 \pi$, we find $z_{e}^{2 D} \cong 0.8206 l$. These numbers are almost identical to those obtained from Eq. (15). Thus we can conclude that $z_{e}^{2 D} \cong 0.82 l$. Our result is noticeably larger than the previously used value $z_{e}^{2 D}=\pi l / 4$ $\cong 0.785 l$ obtained by an analytical approach.

Finally, as a test of our numerical approach in the determination of the extrapolation length, we have also performed 


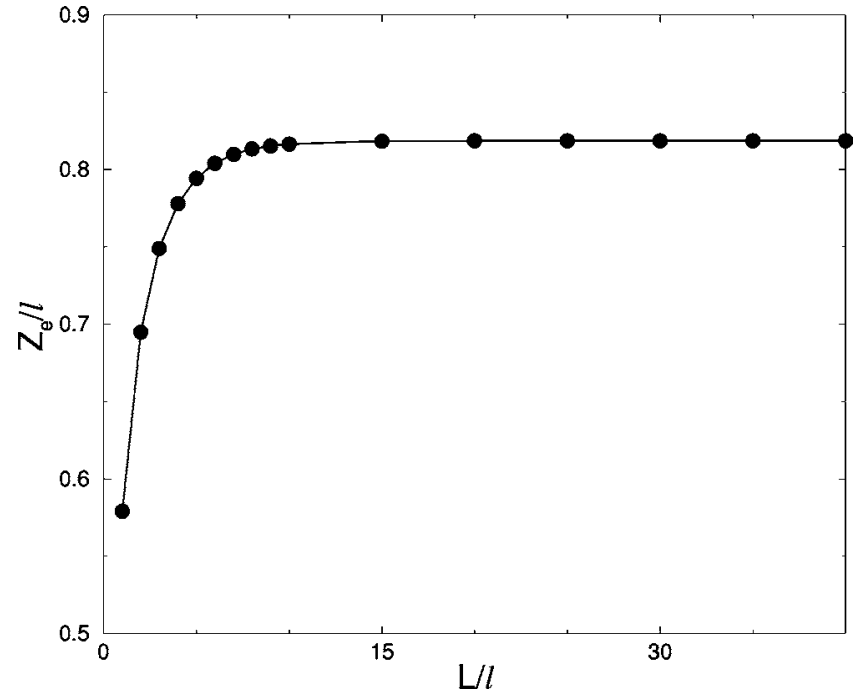

FIG. 7. Obtained $z_{e}^{2 D}$ by the method shown in Fig. 6 as a function of slab thickness $L$, under an approximate point source $\delta(z)$ with $k l=20 \pi$.

the similar calculations based on the Bethe-Salpeter equation for 3D slabs [14]. In Fig. 8 we plot the numerical results of $1 / I(L)$ with a plane wave incidence on a $3 \mathrm{D}$ slab of thickness $L$. From which we find $z_{e}^{3 D} \cong 0.7112 l$. This number is very close to $0.7104 l$ obtained from the Milne solution. This shows the reliability of our $2 \mathrm{D}$ results presented here.

\section{CONCLUSION}

In this work, we have studied the wave transporting behavior through a $2 \mathrm{D}$ random slab by using a first-principles approach based on the Bethe-Salpeter equation. A general 2D vertex function for the ladder diagrams has been derived from the Ward identity. This allows us to calculate correctly the static and dynamic transmitted intensities. When the slab thickness $L$ is comparable to the mean free path $l$, we found an abrupt crossover from the ballistic to diffusive behavior

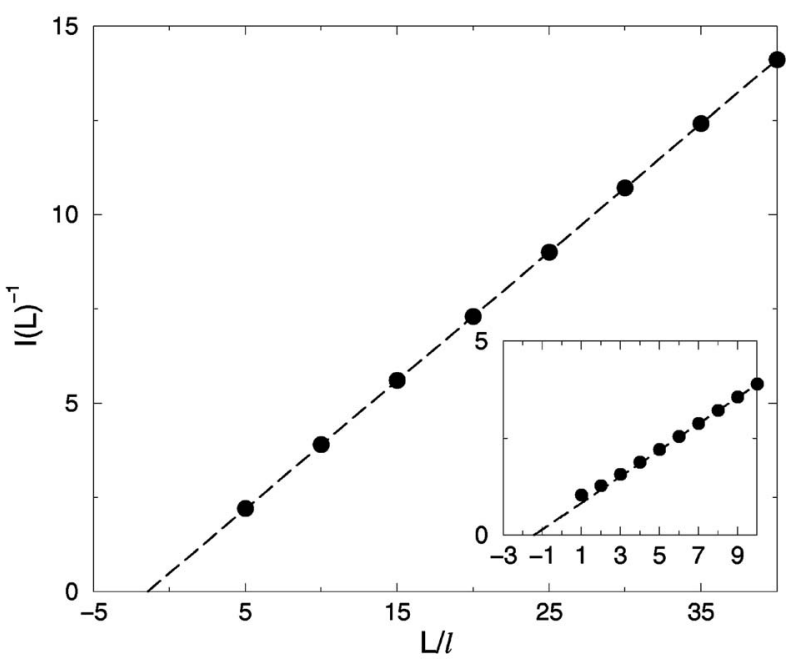

FIG. 8. The inverse of scattered intensity $I(L)^{-1}$ in $3 \mathrm{D}$, as a function of the slab thickness $L$. The dashed line is the linear fit of data for large $L$. The inset graph shows data for $L \leqslant 5 l$.

when $L_{c} \cong 6 l$. This number is much larger than $L_{c} \cong 3 l$ found in $3 \mathrm{D}$. In the transition region, the ballistic-like peak and the peak of diffusive waves coexist in the time-domain profile. This is consistent with the experimental observation in ultrasonic measurements. In the diffusive regime, when $L \gg l$, we have studied the extrapolation length and obtained $z_{e}^{2 D}$ $\cong 0.82 l$ in $2 \mathrm{D}$, which is noticeably larger than the previously used value $\pi l / 4$ obtained by analytical approach. The above results are insensitive to the choice of the center frequency as long as the localization length is much greater than the slab thickness.

\section{ACKNOWLEDGMENT}

This work was supported by Hong Kong RGC Grant No. 604703.
[1] See, for example, Scattering and Localization of Classical Waves in Random Media, edited by P. Sheng (World Scientific, Singapore, 1990); P. Sheng, Introduction to Wave Scattering, Localization and Mesoscopic Phenomena (Academic Press, New York, 1995); M. C. W. van Rossum and T. M. Nieuwenhuizen, Rev. Mod. Phys. 71, 313 (1999); A. Lagendijk and B. A. van Tiggelen, Phys. Rep. 270, 143 (1996).

[2] Y. Kuga and A. Ishimaru, J. Opt. Soc. Am. A 1, 831 (1984); M. P. Van Albada and A. Lagendijk, Phys. Rev. Lett. 55, 2692 (1985); P. E. Wolf and G. Maret, ibid. 55, 2696 (1985); E. Akkermans, P. E. Wolf, and R. Maynard, ibid. 56, 1471 (1986); M. Kaveh, M. Rosenblush, I. Edrei, and I. Freund, ibid. 57, 2049 (1986).

[3] J. H. Li, A. A. Lisyansky, T. D. Cheung, D. Livdan, and A. Z. Genack, Europhys. Lett. 22, 675 (1993).

[4] J. H. Page, H. P. Schriemer, A. E. Bailey, and D. A. Weitz,
Phys. Rev. E 52, 3106 (1995)

[5] E. Wolf, Phys. Rev. Lett. 56, 1370 (1986); B. Shapiro, ibid. 57, 2168 (1986); M. Stephen and G. Cwilich, ibid. 59, 285 (1987); S. Feng, C. Kane, P. A. Lee, and A. D. Stone, ibid. 61, 834 (1988); I. Freund, M. Rosenbluh, and S. Feng, ibid. 61, 2328 (1988); N. Garcia and A. Z. Genack, ibid. 63, 1678 (1989); R. Berkovits, M. Kaveh, and S. Feng, Phys. Rev. B 40, 737 (1989); M. P. van Albada, J. F. de Boer, and A. Lagendijk, Phys. Rev. Lett. 64, 2787 (1990).

[6] D. A. Weitz, D. J. Pine, P. N. Pusey, and R. J. A. Tough, Phys. Rev. Lett. 63, 1747 (1989); I. Freund, M. Kaveh, and M. Rosenbluh, ibid. 60, 1130 (1988).

[7] R. H. J. Kop, P. de Vries, R. Sprik, and A. Lagendijk, Phys. Rev. Lett. 79, 4369 (1997).

[8] K. M. Yoo, F. Liu, and R. R. Alfano, Phys. Rev. Lett. 64, 2647 (1990). 
[9] K. M. Yoo and R. R. Alfano, Opt. Lett. 15, 320 (1990).

[10] F. C. MacKintosh and S. John, Phys. Rev. B 40, 2383 (1989).

[11] P.-A. Lemieux, M. U. Vera, and D. J. Durian, Phys. Rev. E 57, 4498 (1998).

[12] A. A. Chabanov and A. Z. Genack, Phys. Rev. E 56, R1338 (1997).

[13] A. Garcia-Martin, J. J. Saenz, and M. Nieto-Vesperinas, Phys. Rev. Lett. 84, 3578 (2000).

[14] Z. Q. Zhang, I. P. Jones, H. P. Schriemer, J. H. Page, D. A Weitz, and P. Sheng, Phys. Rev. E 60, 4843 (1999); Z. Q Zhang and P. Sheng, in Photonic Band Gap Materials, Vol. 315 of NATO Advanced Study Institute, Series E: Applied Sciences, edited by C. M. Soukoulis (Kluwer Academic, Dordrecht, 1996).

[15] X. Zhang and Z. Q. Zhang, Phys. Rev. E 66, 016612 (2002).

[16] A. Tourin, A. Derode, A. Peyre, and M. Fink, J. Acoust. Soc. Am. 108, 503 (2000).
[17] A. Tourin, M. Fink, and A. Derode, Waves Random Media 10, R31 (2000)

[18] G. Placzek and W. Seidel, Phys. Rev. 72, 550 (1947).

[19] J. X. Zhu, D. J. Pine, and D. A. Weitz, Phys. Rev. A 44, 3948 (1991).

[20] M. Haney and R. Snieder, Phys. Rev. Lett. 91, 093902 (2003).

[21] E. Abrahams, P. W. Anderson, D. C. Licciardello, and T. V. Ramakrishnan, Phys. Rev. Lett. 42, 673 (1979).

[22] B. C. Gupta and Z. Ye, Phys. Rev. E 67, 036606 (2003).

[23] A. A. Asatryan, P. A. Robinson, R. C. McPhedran, L. C. Botten, C. Martijn de Sterke, T. L. Langtry, and N. A. Nicorovici, Phys. Rev. E 67, 036605 (2003).

[24] K. van Wijk, M. Haney, and J. A. Scales, Phys. Rev. E 69, 036611 (2004).

[25] M. B. van der Mark, M. P. van Albada, and A. Lagendijk, Phys. Rev. B 37, 3575 (1988).

[26] R. Berkovits and S. Feng, Phys. Rep. 238, 135 (1994). 